\section{Epidermoid oder Arachnoidalzyste: CISS, FLAIR und Diffusionsbilder als Ausweg aus dem diagnostischen Dilemma}

\begin{abstract}
Die 45-jährige Patientin erlitt zwei Jahre vor der Vorstellung in unserer Klinik eine Trigeminusneuralgie rechts. Die auswärts durchgeführte MR-Tomographie zeigte in der $\mathrm{T}_{2}$-Gewichtung eine liquorisointense Raumforderung im Kleinhirnbrückenwinkel, die als Arachnoidalzyste gedeutet wurde. Die operative Entlastung dieser Raumforderung führte nur kurzzeitig zu einer Besserung der Symptomatik. Die Patientin stellte sich jetzt bei uns vor, um die Frage einer erneuten Entlastung ihrer „Arachnoidalzyste“ abzuklären.
\end{abstract}

Die alten auswärtig erstellten MR-Tomogramme (Abb.1a) ließen differenzialdiagnostisch an eine Arachnoidalzyste oder an ein typisch lokalisiertes Epider- b moid im Kleinhirnbrückenwinkel auf der rechten Seite denken. Die jetzt durchgeführte MR-Tomographie zeigte in den $\mathrm{T}_{1}$ - und $\mathrm{T}_{2}$-gewichteten Sequenzen wiederum eine praktisch liquorisointense Raumforderung im Kleinhirnbrückenwinkel rechts, die zu einer erheblichen Ausspannung des Nervus trigeminus führte. Die während der gleichen Untersuchung erstellten CISS - (constructive interference in steady state) und diffusionsgewichteten Aufnahmen ließen dann den Tumor eindeutig vom Liquor abgrenzen. In der CISS-Sequenz zeigte sich der Tumor deutlich signalgemindert im Vergleich zum umgebenden Liquor und in seiner gesamten Ausdehnung (Abb.1 b). Er reichte vom kraniozervikalen Übergang nach kranial fast bis an das Tentorium heran und ventral bis an den Austrittspunkt des Nervus oculomotorius aus dem Hirnstamm. Auf Höhe des Austrittspunktes des achten Hirnnerven zeigte sich innerhalb des Tumors ein Defekt mit liquorisointensem Signal, der einem postoperativen Defektzustand im Tumor entsprechen kann oder aber mit einem lobulierten Epidermoid vereinbar wäre. In der diffusionsgewichteten Sequenz und in der FLAIR (fluid-attenuated inversion recovery)-Sequenz hatte der

Fortschr Röntgenstr 2001; 173: 77-78 (C) Georg Thieme Verlag Stuttgart · New York ISSN 1438-9029 abgrenzen (Abb. $1 \mathbf{c}$ und $\mathbf{1} \mathbf{d}$ ).

\section{Diskussion}
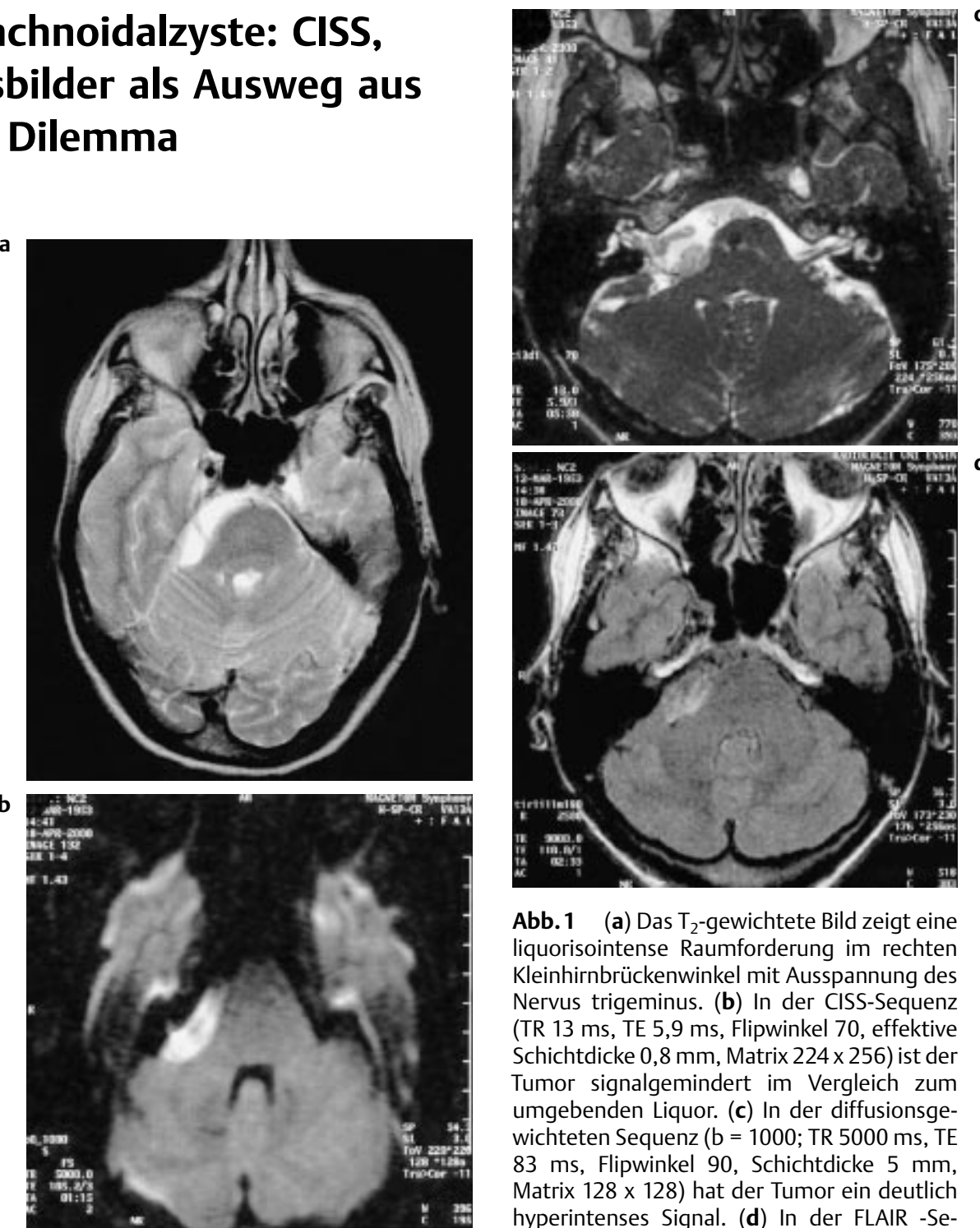

Abb.1 (a) Das $T_{2}$-gewichtete Bild zeigt eine liquorisointense Raumforderung im rechten Kleinhirnbrückenwinkel mit Ausspannung des Nervus trigeminus. (b) In der CISS-Sequenz (TR 13 ms, TE 5,9 ms, Flipwinkel 70, effektive Schichtdicke $0,8 \mathrm{~mm}$, Matrix $224 \times 256$ ) ist der Tumor signalgemindert im Vergleich zum umgebenden Liquor. (c) In der diffusionsgewichteten Sequenz ( $b=1000$; TR 5000 ms, TE $83 \mathrm{~ms}$, Flipwinkel 90, Schichtdicke $5 \mathrm{~mm}$, Matrix $128 \times 128$ ) hat der Tumor ein deutlich hyperintenses Signal. (d) In der FLAIR -Sequenz stellt sich der Tumor hyperintens dar und ist klar vom hypointensen Liquor abgrenzbar.

Tumor ein deutlich hyperintenses Signal und ließ sich ebenfalls klar vom Liquor

Epidermoide entstehen aus intrakraniellen Einschlüssen von epithelialen Zellen während des Neuralrohrschlusses und sind etwa 10-fach häufiger als intrakranielle Dermoide. Sie haben nur eine geringe Wachstumstendenz und werden deshalb oft erst im frühen Erwachsenenalter symptomatisch. Am häufigsten sind intrakranielle Epidermoide im Kleinhirnbrückenwinkel lokalisiert (50\%), sellär und parasellär (10-15\%), seltener im vierten Ventrikel und in der präpontinen Zisterne. Etwa 10\% der
Epidermoide kommen intraossär im Schädelknochen vor.

Arachnoidalzysten liegen zu 50\% in der Nähe der Sylvischen Fissur und zu 20\% in der suprasellären Zisterne. Immerhin $15-20 \%$ der Arachnoidalzysten finden sich im Kleinhirnbrückenwinkel, so dass sich die Differenzialdiagnose zwischen Epidermoid und Arachnoidalzyste nicht selten stellt. Einen klinischen Hinweis auf ein Epidermoid gibt die Trigeminusneuralgie. Diese tritt bei ca. $80 \%$ der Epidermoide auf. Das Vorhandensein von Arachnoidalzysten führt im Gegensatz dazu nur äußerst selten zu einer Affektion des N. trigeminus (Koening E et al.; in: Conscientia diagnostica, Kon- 
stanz, Byk Gulden 1987; 8-15). In der CT-Ära galt die Dichtemessung mit Bestimmung der Standardabweichung als einigermaßen verlässlich. In der Regel wurde aber eine Zisternographie mit jodhaltigem Kontrastmittel durchgeführt. Der Dichteanstieg galt als beweisend für die Arachnoidalzyste. Auch heute noch wird dieses Verfahren propagiert, denn die „konventionelle“ MRDiagnostik mit $\mathrm{T}_{1}$ - und $\mathrm{T}_{2}$-gewichteten SE-Sequenzen hat die differenzialdiagnostischen Probleme nicht gelöst (Ikushima I et al.; AJNR 1997; 1359-1363). Unsere Patientin wurde initial unter der Diagnose einer Arachnoidalzyste operiert, weil das MR eine liquorisointense Raumforderung im Kleinhirnbrückenwinkel zeigte.

Auch wenn viele Epidermoide sich in $\mathrm{T}_{1}$ und $\mathrm{T}_{2}$-Wichtung leicht hyperintens darstellen können, bleiben dennoch einige dieser Tumoren problematisch aufgrund ihrer fehlenden Abgrenzbarkeit zum Liquor (Kallmes DF et al.; AJR 1997; 883 -887). Lediglich Epidermoide mit einem hohen Anteil an Proteinen innerhalb der Zyste können ein hyperintenses Signal in der $\mathrm{T}_{1}$-Gewichtung im Vergleich zum Liquor zeigen (Ochi M et al.; AJNR 1998; 1113 -1115) sowie weiterhin ein deutlich hypointenses $\mathrm{T}_{2}$-Signal aufweisen (Timmer et al.; AJNR 1998; 1111 1112).

Die FLAIR-Sequenz ist stark $\mathrm{T}_{2}$-gewichtet, jedoch wird das Liquorsignal zum größten Teil unterdrückt. Dadurch ist eine bessere Abgrenzung des Tumors vom Liquor möglich. Es besteht jedoch der Nachteil von deutlichen Flussartefakten (Ikushima). Weiterhin kommt der Vorteil der FLAIR-Sequenz nur bei wenig wasserhaltigen Tumoren zum Tragen; Tumoren mit hohem Wasseranteil werden in ihrem Signal ebenso wie der Liquor unterdrückt und sind damit wieder isointens (Ikushima).

Diffusionsgewichtete Bilder stellen die Bewegung von Wassermolekülen dar. Auch diese Sequenz eignet sich somit zur Differenzierung zwischen soliden Strukturen und dem Liquor; solide Strukturen wie Epidermoide zeigen einen Anstieg der Signalintensität (Murakami N et al.; Neurosurg Rev 1999; 159 -162). Von Bedeutung ist hier, dass ein relativ hoher b-Wert und damit eine starke Diffusionswichtung angewendet wird, um die $\mathrm{T}_{2}$-Kontraste $\mathrm{zu}$ minimieren. Als zusätzlicher Parameter können ADC-
Maps dienen. Epidermoide zeigen einen niedrigen ADC-Wert, der noch etwas niedriger als der Wert von Hirnparenchym liegt. Arachnoidalzysten hingegen haben, ebenso wie Liquor, einen hohen ADC-Wert. In dem hier vorgestellten Fall fand sich ebenfalls eine gute Abgrenzbarkeit des Tumors vom Liquor, das Epidermoid stellte sich in der Diffusionswichtung $(b=1000)$ hyperintens dar (Abb. 1 c).

Die CISS-Sequenz ist eine $\mathrm{T}_{2}$-gewichtee Sequenz und eine Variante der "fast imaging with steady-state precession“ (FISP) mit dem Vorteil einer guten Auflösung. Eine deutliche Differenzierung zwischen Liquor und soliden Strukturen ist möglich (Ikushima). CISS-Bilder zeigen die Epidermoide mit hypointensem Signal gegenüber Liquor. Durch die Möglichkeit von 3D-Rekonstruktionen verbessert sich die räumliche Zuordnung.

Zur genauen Bestimmung der Ausbreitung erwies sich im vorgestellten Fall die CISS-Sequenz als besonders hilfreich; das Epidermoid stellte sich deutlich hypointens zum Liquor dar. Durch die dünnen Schichten $(0,7 \mathrm{~mm})$ kommen die exakte Größe und die Beziehung zu anatomischen Strukturen wie dem N. vestibulocochlearis klar zur Darstellung (Abb.1 b).

Das differenzialdiagnostische Dilemma: Epidermoid oder Arachnoidalzyste bei Raumforderungen im Kleinhirnbrückenwinkel kann mit Hilfe der modernen MRT gelöst werden. Die Anwendung von CISS, FLAIR oder Diffusionssequenzen erlaubt eine sichere Unterscheidung und macht die CT-Zisternographie überflüssig.

E. R. Gizewski, Essen 